Journal of Engineering and Science Research 2 (1): 01-05, 2018

e-ISSN: 2289-7127

(C) RMP Publications, 2018

DOI: $10.26666 /$ rmp.jesr.2018.1.1

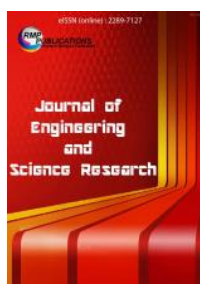

\title{
Combining Docking and Molecular Dynamic of Protease from Bacillus lehensis G1
}

\author{
Noorul Aini Sulaiman ${ }^{1,2}$, Shuhaila Mat-Sharani ${ }^{2}$, Nor Muhammad Mahadi $^{2}$ and Nur Zazarina Ramly ${ }^{1}$ \\ ${ }^{1}$ Food Biotechnology, Faculty of Science and Technology, Islamic Science University of Malaysia \\ (USIM), Bandar Baru Nilai 71800, Nilai, Negeri Sembilan, Malaysia \\ ${ }^{2}$ Malaysia Genome Institute, Jalan Bangi, 43000 Kajang. Selangor, Malaysia School of Mechanical
}

\begin{abstract}
Protease is an enzyme that catalysed the hydrolysis of protein into peptide. Application of protease in industry has been linked with cost effective substrates and complex of enzyme-substrate stability. Molecular docking approach has identified casein as a preference substrates. However, lack of data on casein mode of binding to protease and enzyme stability represents a limitation for its production and structural optimization. In this study, we have used a molecular dynamic (MD) to examine the stability of complex enzyme-substrate of protease from Bacillus lehensis G1. The 3D structure of protease (BleG1_1979) was docked with substrate casein using AutoDock Vina. Structural analysis of the substrate-binding cleft revealed a binding site of casein was predominantly at the hydrophobic region of BleG1_1979. The MD of complex BleG1_1979-casein was tested with two temperatures; $298 \mathrm{~K}$ and $310 \mathrm{~K}$ using GROMACS v5.1.4. MD simulation showed a stable behaviour of BleG1_1979 over the 20 ns simulation period. The molecular docking and MD simulation suggested that the production of protease from $B$. lehensis G1 by utilization of casein and the stability of complex protease-casein could be a potential application to generate a cost effective enzyme to be develop for industrial use
\end{abstract}

Key words: Bacillus lehensis G1, protease, molecular docking, molecular dynamic

\section{INTRODUCTION}

Proteases is an enzyme that belong to the hydrolases group that is mostly extracellular [1]. Proteases involves in various function includes nutrient digestion, proteins catabolism, protein turnover, hydrolysis abnormal protein, and etc. [2]. In human, protease is exceptionally important signalling molecules that involved in various vital processes through strictly regulated pathways. The dysregulation of protease in our body may cause illness such as cardiovascular, inflammatory diseases, cancer, osteoporosis and neurological disorders [3]. Whilst, in microorganism, protease enzyme are known to provide peptide nutrients to bacteria. Though, this enzyme could as well mediate damage to host tissue in pathogenic microorganism [4].

Microbial proteases are important industrially, however only a few are recognized as commercial producers, such as subtilisin Carlsberg, subtilisin BPN' and Savinase [5]. Various study has been carried out on manipulation of microbial protease for industrial application such as detergent, nutrition, medical, hide dehairing agent, bio-waste removal, silk degumming, and recovery of silver from X-ray/photographic films [6-11]. The development of recombinant protease using protein engineering to enhance the properties of enzyme for maximal production has been successfully carried out. For example, triple mutation on protease from $B$. pumilus CBS exhibit the highest catalytic efficiency and higher degree of peptide hydrolysis when express recombinant in B. subtilis DB430 [12].

By using in silico technique, an enzyme properties could be discovered beforehand prior to massive production in laboratories or industrially. Molecular docking is a computational technique that predict noncovalent binding of a small molecule onto macromolecules with aim to predict the bound conformations and the binding affinity [13]. Whilst, MD simulation is a tool in bioinformatic approach that

Corresponding Author: Noorul Aini Sulaiman, USIM, Faculty of Science and Technology, Islamic Science University of Malaysia, Bandar Baru Nilai Negeri Sembilan, Malaysia, +60102141941. 
provide a spatial and temporal resolution that is not available in the laboratories experiments. MD simulations have become more precise with improved force fields, motions on the micro-second, and scale processes such as protein folding [14]. In this study, we will report MD simulation of docked B. lehensis G1 BleG1_1979 protease with casein. In an effort to elucidate the stability of the complex BleG1_1979casein in MD, the RMSD and RMSF generated from GROMACS v5.1.4 were analysed in details.

\section{EXPERIMENT}

\section{Molecular Docking}

The 3D structure of BleG1_1979 was built previously using Modeller v9.18 [15-16]. The docking of BleG1_1979 with casein was carried out using AutoDock Vina v1.1.2 [17]. Casein structure were retrieved from PubChem website (https://pubchem.ncbi.nlm.nih.gov/). Analysis of interaction in AutoDock Vina was carried out using MGLTools (v1.5.6) [18].

\section{Molecular Dynamic}

The docked BleG1_1979-casein structure was simulated using the GROMACS v5.1.4 [19] simulation software to examine the structural stability at two temperatures which are $298 \mathrm{~K}$ and $310 \mathrm{~K}$. BleG1_1979-casein was solvated in a box of explicit simple point charge (SPC) water molecules and simulated using periodic boundary conditions (PBC) and particle mesh Ewald (PME) summation to improve electrostatic interactions. Structures were minimized using 200 steps from the steepest descent method.

\section{RESULT AND DISCUSSION}

The 3D structure of BleG1_1979 from B. lehensis G1 was generated using Modeller v9.18 was docked with casein structure. Computational docking result showed that casein probably binds to BleG1_1979 at the hydrophobic region. The molecular docking was carried out to recognize the binding site and the best binding conformation of casein to BleG1_1979. Autodock Vina showed the best pose of casein with the binding energy of $-5.6 \mathrm{kcal} / \mathrm{mol}$. A details observed of the binding site showed two hydrogen bond interactions between the amide group of the Gly146 from BleG1_1979 with carbonyl group of Pro7 and Pro8 from casein (Table 1). The nitrogen backbone from Gly146 forms a bifurcated hydrogen bond with the oxygens of Pro7 and Pro8 with interatomic distances of $2.498 \AA$ and $2.396 \AA$, respectively (Figure 1a). Another hydrogen bond was form between Glu11 from casein with Lys162 from BleG1_1979 with distance of $2.326 \AA$ (Figure 1b).

Further analysis of casein binding with BleG1_1979 showed lack of pi-pi and pi-cation interaction. In total, substrate casein forms three hydrogen bonds with BleG1_1979. Summary of analysis from MGLTools (v1.5.6) showed hydrogen bond and van der Waals contact between the residues in the BleG1_1979 with casein in spheres wireframe as showed in Figure 2. The van der Waals interaction involves hydrophobic residues such as Thr140, Ala141, Val142, Ile148, Ile149, Ala163, and Gly144. Whilst, the charges residues are Asn119, Lys167, and Asp106. Hence, the docking results suggested the binding of casein onto BleG1_1979 are mostly involves hydrophobic residues.

(a)

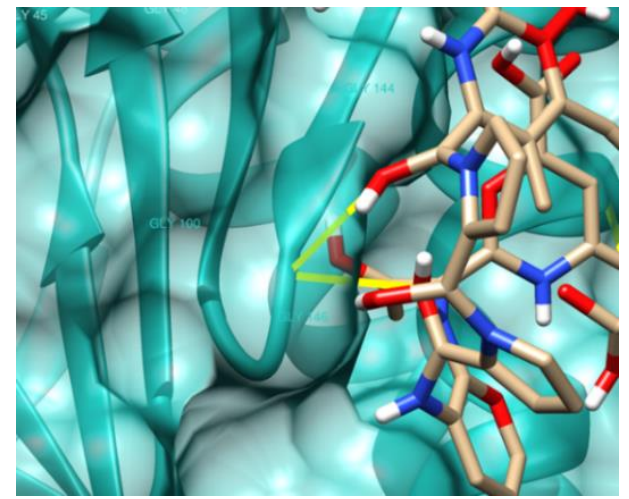

(b)

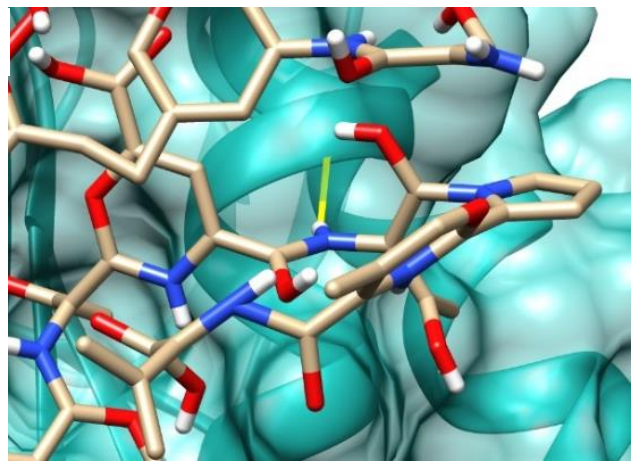

Figure 1 Binding interaction between substrate casein with BleG1_1979 with lowest energy $-5.6 \mathrm{kcal} / \mathrm{mol}$. (A) Two hydrogen bonds formation from Gly146 BleG1_1979, (B) Single hydrogen bond between Glu11 in casein with Lys162 in BleG1_1979. The image is generated using Chimera v1.11.2 as BleG1_1979 is showed as ribbon structure with $60 \%$ transparency of electrostatic surface and casein is showed as stick figure.

Table 1 Hydrogen bonds between residues of BleG1_1979 and casein in molecular docking

\begin{tabular}{ccccc}
\hline $\begin{array}{c}\text { BleG1_1979 } \\
\text { residues }\end{array}$ & Atom & $\begin{array}{c}\text { Casein } \\
\text { residues }\end{array}$ & Atom & Distance \\
\hline Gly146 & NH & Pro7 & O & $2.498 \AA$ \\
Gly146 & NH & Pro8 & O & $2.398 \AA$ \\
Lys162 & OH & Glu11 & H & $2.326 \AA$ \\
\hline
\end{tabular}




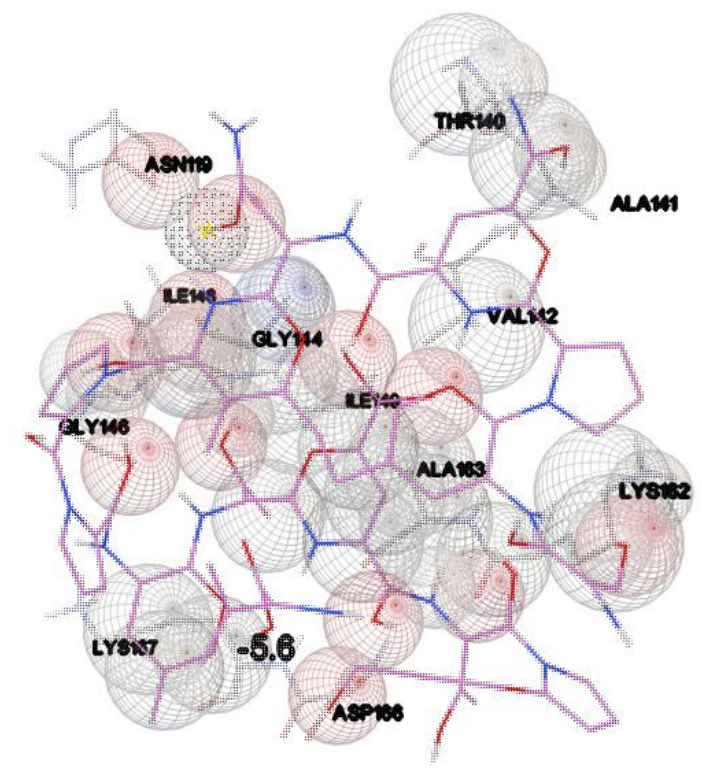

Figure 2 van der Waals contact between casein and BleG1_1979. The sphere wireframe showed van der Waals contact and yellow dot showed hydrogen bond. The image is generated using AutoDock Tools from MGLTools (v1.5.6) software package.

The complex of BleG1_1979-casein was simulated using the GROMACS v5.1.4 simulation software to examine the structural stability at two different temperatures; the room temperature of $298 \mathrm{~K}$ $\left(25^{\circ} \mathrm{C}\right)$ and the growth optimum temperature $310 \mathrm{~K}$ $\left(37^{\circ} \mathrm{C}\right)$ of $\mathrm{B}$. lehensis $\mathrm{G} 1$. Simulation at these different temperatures is expected to show a comparable MD stability profile. BleG1_1979 was solvated in a box of explicit simple point charge (SPC) water molecules and simulated using periodic boundary conditions (PBC) and particle mesh Ewald (PME) summation to improve electrostatic interaction. The docked structures were minimized using 200 steps from the steepest descent method. The systems were then subjected to MD simulations for $20 \mathrm{~ns}$. LINCS algorithm was used to constrain bond length with a time step of 10 fs for all calculations. Long range electrostatic interactions were calculated using Particle Mesh Ewald (PME) with cubic interpolation. van der Waals and Coulombic interactions were truncated at $1 \mathrm{~nm}$. The nonbonded pair list was updated every 5000 steps and conformations were stored every $10 \mathrm{ps}$ during simulations. Protein structure were further visualize using VMD [20-21]. The stability and flexibility of structure were count in backbone root mean square deviations (RMSD) and root mean square fluctuation (RMSF) and view using grace (http://plasma-gate.weizmann.ac.il/Grace/).

The complex of BleG1_1979-casein was analysed for their stabilization and flexibility of the complex structure. RMSD analysis of the $\mathrm{C} \alpha$ value showed that the complex enzyme-substrate was stable at both temperature as showed in Figure 3. Whilst, the dynamics of residues from the complex structure was analysed using RMSF. RMSF results showed a high fluctuations for the coils structure for both temperature tested. The RMSF of the BleG1_1979-casein at $298 \mathrm{~K}$ was comparable with $310 \mathrm{~K}$, except a higher fluctuation value was observed at residues 30-50 and 90-100, respectively (Figure 4). At $298 \mathrm{~K}$, RMSF displayed the highest fluctuation at $0.45 \mathrm{~nm}$ and these coils region were indicated for as flexible was shown in figure 5. From our MD data, the complex of BleG1_1979-casein showed an increased conformational flexibility at Gly, Tyr, Ile, Lys, Val, and Pro on coils structure. This coils structure could possibly be essential to enable transport of casein into the catalytic site where Cys106 are situated in the hydrophobic region. Coils are significantly important structure that may have play a vital parts in recognition, ligand binding or forming an enzyme active sites [22]. According to HenzlerWildman et al. [23], enzymatic activities require a particular balance between flexibility and stability. Enzymes must be stable to retain their 3D structures, but at the same time, sufficiently flexible to allow for substrate binding, chemical reaction and releasing of product to function. Thus, the interaction between BleG1_1979 with casein and their dynamics is important to carried out the role of enzyme catalysis.

The conformational changes of enzymes are often related to regulating and creating an optimal environment for efficient chemical catalysis. The dynamics and conformational changes in enzymes may range from the fluctuation of side chains to the largescale protein motions. The fluctuation helps regulating the environment for chemical reactions and the large motion allows the protein to create a tertiary structure for substrate binding. The internal motions of proteins might assist as 'gate' in some systems, which controls ligand-protein association. In many enzymes or complexes, protein motions involve an allosteric communication to coordinate the function and reactions, which could be intrinsic to most enzymes [24]. 


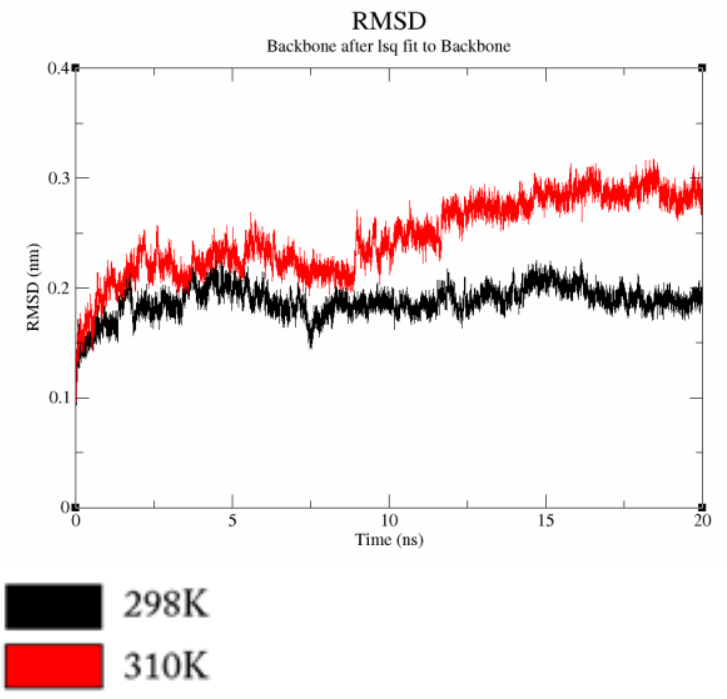

Figure 3 A RMSD analysis as for complex BleG1_1979-casein as a function of time at $298 \mathrm{~K}\left(25^{\circ} \mathrm{C}\right)$ and $310 \mathrm{~K}\left(37^{\circ} \mathrm{C}\right)$. BleG1_1979 simulated at $298 \mathrm{~K}$, displayed a more consistent RMSD value compared to the structures simulated at $310 \mathrm{~K}$.

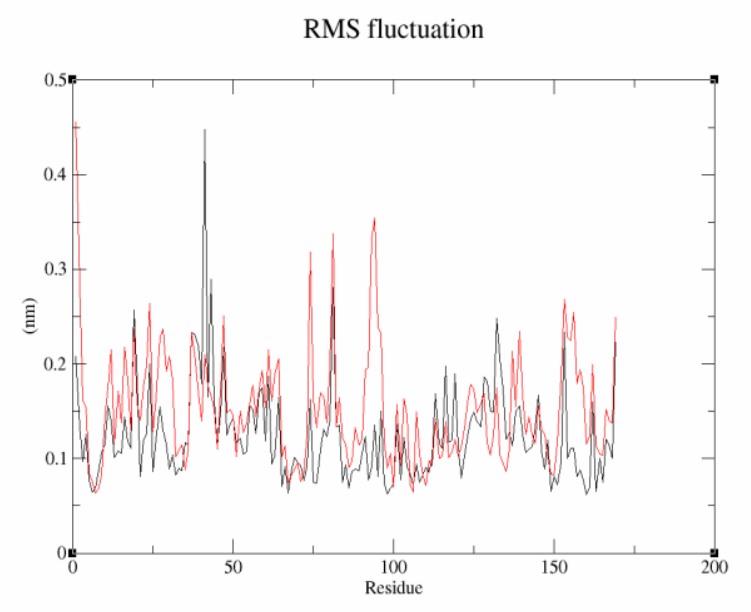

$298 \mathrm{~K}$

$310 \mathrm{~K}$

Figure 4 RMSF of the C $\alpha$ atom of complex BleG1_1979casein at $310 \mathrm{~K}$. The structure simulated at $310 \mathrm{~K}$ showed different residues fluctuations than the structure simulated at $298 \mathrm{~K}$.

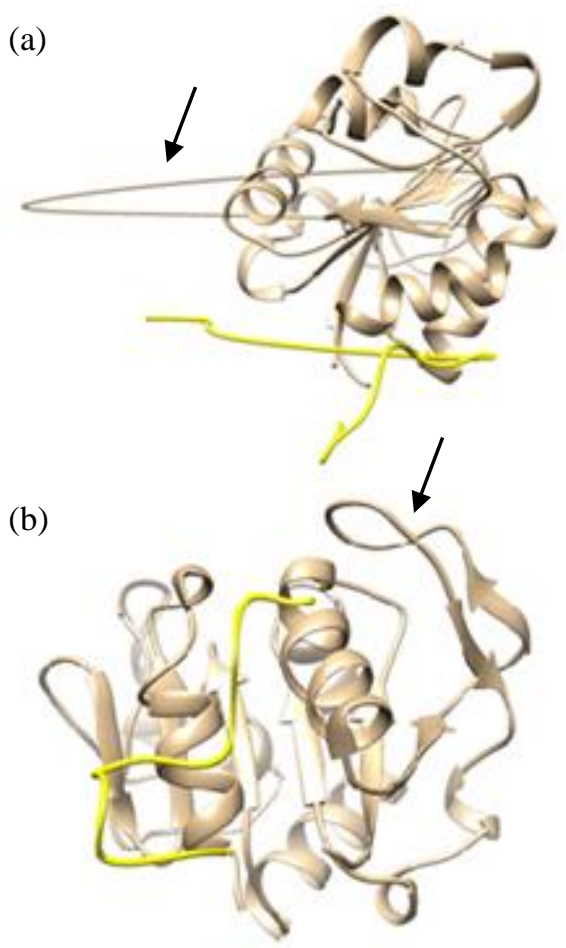

Figure 5 Docked complex of BleG1_1979-casein showing position of flexible loops structure at (A) $298 \mathrm{~K}$ and (B) 310 K.

Proteins are usually functions by administered of their dynamical character. Any modification of protein dynamics are believed to effect in molecular adaptation of enzymes at different temperatures. An evolutionary pressure in temperature variation seems to be on preserving and optimizing functionally important motions. For instance, a cold adapted enzymes are thought to have higher structural flexibility, from their high catalytic rates at lower temperatures, as compared to meso- and thermophilic organisms, but with a lower thermal stability. Whilst, enzymes from thermophiles seems to be more rigid, conformationally stable protein structures, which are reflected in lower catalytic activity at lower temperatures. However, the relationship between catalytic activity, thermal stability and molecular flexibility is vastly complex and the fundamental principles are still poorly understood [25].

\section{CONCLUSION}

The present study of molecular docking showed casein has form three hydrogen bonds and several van der Waals contact with protease BleG1_1979 from $B$. lehensis G1. Whilst, the MD simulation showed the stability and the flexibility of coils in the complex of enzyme-substrate. This result suggests that the protease BleG1_1979 from B. lehensis G1 might cleave casein and form a stable structure. Thus, this study provide a 
Noorul Aini Sulaiman/Journal of Engineering and Science Research, 2(1) 2018, Pages: 01-05

preliminary data on interaction in complex enzymesubstrate from bacterial source that could be useful for production of protease using an inexpensive substrate such as casein.

\section{ACKNOWLEDGMENT}

The authors would like to thank Science Fund from the Malaysia Ministry of Science, Technology and Innovation (MOSTI) (Grant Number: USIM/SF-UKM/FST/30/40214) and Malaysia Genome Institute for financial support and computer resources, respectively.

\section{REFERENCES}

[1] Illanes, A. 2008. Enzyme biocatalysis: principles and applications. Springer.

[2] Rao, M. B., Tanksale, A. M., Ghatge, M. S. and Deshpande, V. V. 1998. Molecular and biotechnological aspects of microbial proteases. Microbiology and molecular biology reviews 62(3), 597-635.

[3] Turk, B. 2006. Targeting proteases: successes, failures and future prospects. Nature reviews drug discovery 5(9), 785-99.

[4] Lantz, M. S. 1997. Are bacterial proteases important virulence factors?. Journal of Periodontal Research 32, 126-132.

[5] Gupta, R., Beg, Q. K. and Lorenz, P. 2002. Bacterial alkaline proteases: molecular approaches and industrial applications. Applied microbiology and biotechnology 59, 15-32.

[6] Haddar, A., Sellami-Kamoun, A., Fakhfakh-Zouari, N., Hmidet, N. and Nasri, M. 2010. Characterization of detergent stable and feather degrading serine proteases from Bacillus mojavensis A21. Biochemical engineering journal 51, 53-63.

[7] Bajaj, B. K., Sharma, N. and Singh, S. 2013. Enhanced production of fibrinolytic protease from Bacillus cereus NS-2 using cotton seed cake as nitrogen source. Biocatalysis and agricultural biotechnology 2(3), 204209.

[8] Sundararajan, S., Kannan, C. N. and Chittibabu, S. 2011. Alkaline Protease from Bacillus cereus VITSN04: potential application as a dehairing agent. Journal of bioscience and bioengineering 111(2), 128133.

[9] Sinha, R. and Khare, S. K. 2013. Characterization of detergent compatible protease of a halophilic Bacillus Sp. EMB9: differential role of metal ions in stability and activity. Bioresource technology 145, 357-361.

[10]Joshi, S. and Satyanarayana, T. 2013. Characteristics and applications of a recombinant alkaline serine protease from a novel bacterium Bacillus lehensis. Bioresource technology 131, 76-85.

[11]Wang, S.L. and Yeh, P. Y. 2006. Production of a surfactant- and solvent-stable alkaliphilic protease by bioconversion of shrimp shell wastes fermented by Bacillus subtilis TKU007. Process biochemistry 41, 1545-1552.
[12] Jaouadi, N. Z., Jaouadi, B., Aghajari, N. and Bejar, S. 2012. The overexpression of the SAPB of Bacillus pumilus CBS and mutated sapB-L31I/T33S/N99Y alkaline proteases in Bacillus subtilis DB430: new attractive properties for the mutant enzyme. Bioresource technology 105, 142-151.

[13] Trott, O. and Olson, A. 2010. AutoDock Vina: improving the speed and accuracy of docking with a new scoring function, efficient optimization, and multithreading. J Comp Chem 31, 455-461.

[14] Abraham, M.J., Murtola, T., Schulz, R., Smith, J. C., Hess, B. and Lindahl, E. 2015. GROMACS : high performance molecular simulations through multi-level parallelism from laptops to supercomputers. SoftwareX $1-2,19-25$.

[15] Webb, B. and Sali, A. 2014. Comparative protein structure modeling using modeller. Current protocols in bioinformatics. John Wiley \& Sons, Inc. New Jersey. USA.

[16] Sulaiman, N.A., Mahadi, N.M. and Ramly, N.Z. 2017. Identification of proteolytic genes from Bacillus Lehensis G1. Journal of engineering and science research 1(2), 14-20.

[17] Seeliger, D. and de Groot, B.L. 2010. Ligand docking and binding site analysis with PyMOL and Autodock/Vina. Journal of computer-aided molecular design 24(5), 417-422.

[18] Morris, G. and Huey, R. 2009. Autodock4 and Autodocktools4: automated docking with selective receptor flexibility. J comput chem 16, 2785-2791.

[19] van Der Spoel, D., Lindahl, E., Hess, B., Groenhof, G., Mark, A.E. and Berendsen, H.J.C. 2005. GROMACS: fast, flexible, and free. Journal of computational chemistry 26(16), 1701-1718.

[20] Humphrey, W., Dalke, A. and Schulten, K. 1996. VMD: visual molecular dynamics. J mol graph 14(1), 33-38.

[21] Eargle, J., Wright, D. and Luthey-Schulten, Z. 2006. Multiple alignment of protein structures and sequences for VMD. Bioinformatics 22(4), 504-506.

[22] Minuchehr, Z. and Goliaei, B. 2005. Propensity of amino acids in loop regions connecting beta-strands. Protein and peptide letters 12, 379-382.

[23] Henzler-Wildman, K. A., Lei, M., Thai, V., Kerns, S. J., Karplus, M. and Kern, D. 2007. A hierarchy of timescales in protein dynamics is linked to enzyme catalysis. Nature 450, 913-916.

[24] Chang, C., Huang, Y., Mueller, L. and You, W. 2016. Investigation of structural dynamics of enzymes and protonation states of substrates using computational tools. Catalysts 6(82) 1-21.

[25]Sigtryggsdóttir, Á.R., Papaleo, E., Thorbjarnardóttir, S.H. and Kristjánsson, M.M. 2014. Flexibility of coldand heat-adapted subtilisin-like serine proteinases evaluated with fluorescence quenching and molecular dynamics. Biochimica et biophysica acta 1844, 705712. 